\title{
Étude de la condensation en film laminaire d'une vapeur pure et saturée sur la paroi poreuse d'une plaque inclinée
}

\author{
Rachid Chaynane $^{1}$, Mohamed Asbik ${ }^{1, a}$, Hassane Boushaba $^{1}$, Belkacem Zeghmati ${ }^{2}$ \\ ET AHMED KHMOU ${ }^{3}$ \\ ${ }^{1}$ Laboratoire de Thermodynamique et Énergétique (L.T.E), Faculté des Sciences et Techniques, BP 509, Boutalamine, \\ Errachidia, Maroc \\ 2 Groupe de Mécanique d'Acoustique et d'Instrumentation, Centre des Études Fondamentales (GMAI-CEF), \\ Université de Perpignan, Avenue de Villeneuve, 66860 Perpignan Cedex, France \\ 3 Faculté des Sciences, Avenue Beni M’Hamed, BP 4010, Meknès, Maroc
}

Reçu le 4 avril 2002, accepté le 5 mars 2004

\begin{abstract}
Résumé - L'étude en convection forcée de la condensation en film laminaire d'une vapeur pure et saturée sur une plaque poreuse inclinée par rapport à la verticale, est présentée. Le modèle de Darcy-Brinkman est utilisé pour décrire l'écoulement dans le milieu poreux, tandis que les équations classiques de la couche limite ont été exploitées dans le liquide pur en tenant compte des termes d'inertie et de convection d'enthalpie. Le problème posé a été résolu par voies analytique et numérique. Les résultats sont essentiellement présentés sous forme de l'épaisseur adimensionnelle du film liquide, des profils de vitesse et de température et des coefficients d'échanges thermiques représentés par le nombre de Nusselt. Les résultats obtenus ont été comparés à ceux expérimentaux de Renken et al. [1]. Les effets de différents paramètres influents tels que : l'inclinaison $(\phi)$, la viscosité effective (nombre de Reynolds $R e_{K}$ ), l'épaisseur adimensionnelle du substrat poreux $H^{*}$ et la conductivité thermique adimensionnelle $k^{*}$, sur l'écoulement et les transferts thermiques sont illustrés.
\end{abstract}

Mots clés : Condensation / film laminaire / couche poreuse

\begin{abstract}
Study of laminar film condensation on the wall of an inclined porous plate. A problem of forced convection condensation in a thin porous layer is considered. The flow in the porous region is described by the Darcy-Brinkman model while classical boundary layer equations with inertia and enthalpy terms are used in the pure condensate region. This problem is solved analytically and numerically. The results are essentially presented in the form of the dimensionless film thickness, velocity and temperature profiles within the porous layer and the heat transfer represented by the Nusselt number. The comparison of the present results and those of Renken et al. [1] is carried out. The effects of the inclination $(\phi)$, the effective viscosity (Reynolds number $R e_{K}$ ), the dimensionless thickness of porous coating $H^{*}$ and the dimensionless thermal conductivity $k^{*}$, on the flow and the heat transfer enhancement are also documented.
\end{abstract}

Key words: Condensation / laminar film / porous layer

\section{Introduction}

Les transferts thermiques s'effectuant lors de la condensation dans un milieu poreux ont reçu une attention particulière en raison de leurs diverses applications dans les domaines de l'ingénierie thermique, des équipements électroniques, etc.

\footnotetext{
a Auteur correspondant : m.asbik@caramail.com
}

La majorité des études utilisant une paroi recouverte d'une couche poreuse dans le but d'améliorer les transferts thermiques ont été consacrées au phénomène d'évaporation [2-5]. La présence d'un substrat poreux sur une paroi plane engendre un accroissement du taux de transfert de chaleur comparé à celui observé pour une plaque plane non couverte par une couche poreuse soumise aux mêmes conditions aérauliques et thermiques. Ceci est la conclusion fondamentale tirée par les auteurs des travaux précités. 


\section{Nomenclature}

\begin{tabular}{|c|c|}
\hline$C p$ & 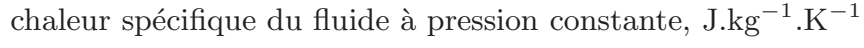 \\
\hline$g$ & accélération gravitationnelle, $\mathrm{m} \cdot \mathrm{s}^{-2}$ \\
\hline$h_{f g}$ & chaleur latente d'évaporation, ${\mathrm{J} . \mathrm{kg}^{-1}}^{-1}$ \\
\hline$H$ & épaisseur de la couche poreuse, m \\
\hline$J a$ & nombre de Jacob \\
\hline$k$ & conductivité thermique, $\mathrm{W} \cdot \mathrm{m}^{-1} \cdot \mathrm{K}^{-1}$ \\
\hline$K$ & conductivité hydraulique, $\mathrm{m}^{2}$ \\
\hline$L$ & longueur de la plaque, $\mathrm{m}$ \\
\hline$N u_{x}$ & nombre de Nusselt local \\
\hline$N u_{L}$ & nombre de Nusselt moyen \\
\hline$(P e)_{\mathrm{eff}}$ & nombre de Peclet \\
\hline $\operatorname{Pr}$ & nombre de Prandtl \\
\hline $\operatorname{Re}_{K}$ & nombre de Reynolds basé sur $\sqrt{K}$ \\
\hline$T$ & température, K \\
\hline$U_{0}$ & vitesse du fluide libre (vapeur), m.s ${ }^{-1}$ \\
\hline$u$ & composante de la vitesse suivant $\mathrm{O} x, \mathrm{~m} . \mathrm{s}^{-1}$ \\
\hline$u_{r}$ & vitesse de référence, $\mathrm{m} . \mathrm{s}^{-1}$ \\
\hline$x$ & abscisse, m \\
\hline$y$ & ordonnée, m \\
\hline \multicolumn{2}{|c|}{ Symboles Grecs } \\
\hline$\alpha$ & diffusivité thermique, $\mathrm{m}^{2} \cdot \mathrm{s}^{-1}$ \\
\hline$\delta$ & épaisseur du film de condensat, m \\
\hline$\varepsilon$ & porosité \\
\hline$\phi$ & angle d'inclinaison par rapport à la verticale, deg $\left(^{\circ}\right)$ \\
\hline$\mu$ & viscosité dynamique, $\mathrm{kg} \cdot \mathrm{m}^{-1} \cdot \mathrm{s}^{-1}$ \\
\hline$\nu$ & viscosité cinématique, $\mathrm{m}^{2} \cdot \mathrm{s}^{-1}$ \\
\hline$\theta$ & température sans dimension \\
\hline$\rho$ & masse volumique, $\mathrm{kg} \cdot \mathrm{m}^{-3}$ \\
\hline \multicolumn{2}{|l|}{ Indices } \\
\hline eff & effective \\
\hline$\ell$ & liquide pur \\
\hline $\mathrm{i}$ & interface substrat poreux/liquide pur \\
\hline $\mathrm{p}$ & milieu poreux \\
\hline $\mathrm{s}$ & saturation \\
\hline $\mathrm{w}$ & paroi \\
\hline$*$ & grandeur adimensionnée \\
\hline
\end{tabular}

Contrairement à l'évaporation, l'étude du processus de condensation dans une couche poreuse couvrant une surface plane n'était pas privilégiée. En effet, Woodruff et al. [6] ont reporté qu'une surface recouverte d'or favorise la condensation en gouttes d'une vapeur à pression atmosphérique. En 1990, Zhao et al. [7] ont utilisé une technique appelée « ion-plating technology » permettant de déclencher la formation de la condensation en gouttes sur une surface couverte d'un substrat poreux. Dans leurs travaux, Shekarriz et al. [7] ont examiné expérimentalement l'effet d'une couche poreuse sur le film de condensat s'écoulant sur la paroi d'un tube horizontal. Ces auteurs ont montré la contribution significative du substrat poreux dans la réduction de l'épaisseur du film liquide et par suite dans l'amélioration des échanges thermiques. 
Cette technique novatrice permettant d'améliorer les transferts de chaleur durant la condensation, a été introduite par Renken et al. [9]. En outre, le même auteur et ses collaborateurs $[1,10-12]$ ont présenté des résultats analytiques et expérimentaux relatifs à la condensation en film laminaire sur une plaque poreuse inclinée. Dans ces références, le modèle de Darcy-Brinkman a été adopté pour décrire l'écoulement dans le milieu poreux. Dans leurs travaux, le rôle significatif du substrat poreux dans l'augmentation du taux de transfert de chaleur par comparaison avec une plaque plane ordinaire (non couverte par une couche poreuse), a été mis en évidence.

Ce travail est consacré à une étude analytique et numérique basée sur les approximations usuelles relatives à un film mince. Le modèle de Darcy-Brinkman est utilisé pour décrire l'écoulement dans le milieu poreux; tandis que les équations classiques de la couche limite ont été exploitées pour décrire les transferts dans le milieu liquide pur. Les conditions de couplage à l'interface milieu poreux/liquide pur, sont essentiellement exprimées par la continuité des contraintes de cisaillement et des densités de flux de chaleur. Les termes d'inertie et de convection d'enthalpie sont négligeables dans la couche poreuse. La résolution numérique est assurée par une méthode aux différences finies implicite. Enfin, nous notons que ce travail fait partie d'une série de travaux que nous avons effectué sur le sujet [13-15].

\section{Analyse}

\subsection{Modèle physique}

Nous nous intéressons au phénomène de la condensation en film laminaire sur une plaque inclinée par rapport à la verticale, recouverte d'un substrat poreux d'épaisseur $H$, de perméabilité $K$ et de porosité $\varepsilon$ (Fig. 1). Cette plaque est placée dans un écoulement de vapeur pure et saturée, de vitesse $U_{0}$. La vapeur se condense sur la paroi de la plaque maintenue à la température $T_{\mathrm{w}}$ inférieure à la température de saturation $T_{\mathrm{s}}$ de la vapeur. Le film de condensat s'écoule sous l'effet de la gravité et des forces de frottement visqueux.

On distingue trois régions : (1) le milieu poreux saturé par le liquide, (2) le film liquide et (3) la vapeur saturante.

Soient $(x, y)$ et $(u, v)$ respectivement les coordonnées Cartésiennes et les composantes de la vitesse du liquide dans le repère associé au modèle.

Nous admettons les hypothèses simplificatrices suivantes :

- Les fluides sont incompressibles.

- La condensation se produit sous forme d'un film mince.

- L'écoulement est permanent, laminaire et bidimensionnel.

- La température à la paroi, les propriétés thermophysiques des fluides et celles de la matrice poreuse sont supposées constantes.
- La viscosité dynamique effective du substrat poreux est égale à celle du film liquide.

- Le substrat poreux est saturé, homogène et isotrope.

- La matrice poreuse est en équilibre local avec le condensat.

- La contrainte de cisaillement à l'interface liquidevapeur est négligeable.

- Généralement, l'épaisseur de la couche poreuse est inférieure à celle du film du condensat.

- La vapeur et le film liquide sont séparés par une frontière distincte.

- Le modèle de Darcy-Brinkman est utilisé pour décrire l'écoulement dans la couche poreuse.

\subsection{Formulation mathématique}

Compte tenu des hypothèses simplificatrices formulées ci-dessus, les équations qui régissent les transferts dans la couche poreuse et le liquide pur ainsi que les conditions aux limites qui leurs sont associées s'écrivent comme dans les sections suivantes.

\subsubsection{Couche poreuse : $0<y<H$}

Dans cette région, le modèle de Darcy-Brinkman (DB) est utilisé pour décrire l'écoulement :

$$
\nu_{\mathrm{eff}} \frac{\partial^{2} u_{\mathrm{p}}}{\partial y^{2}}-\frac{\nu_{\mathrm{eff}}}{K} u_{\mathrm{p}}+g \frac{\rho_{\ell}-\rho_{\mathrm{v}}}{\rho_{\ell}} \cos (\phi)=0
$$

La conservation de la masse et de l'énergie s'expriment par :

$$
\begin{aligned}
& \frac{\partial u_{\mathrm{p}}}{\partial x}+\frac{\partial v_{\mathrm{p}}}{\partial \mathrm{y}}=0 \\
& \frac{\partial^{2} T_{\mathrm{p}}}{\partial y^{2}}=0
\end{aligned}
$$

\subsubsection{Liquide pur : $H<y<\delta$}

Les équations de conservation de la masse, de la quantité de mouvement et de l'énergie s'écrivent :

$$
\begin{aligned}
& \frac{\partial u_{\ell}}{\partial x}+\frac{\partial v_{\ell}}{\partial y}=0 \\
& u_{\ell} \frac{\partial u_{\ell}}{\partial x}+v_{\ell} \frac{\partial u_{\ell}}{\partial y}=\nu_{\ell} \frac{\partial^{2} u_{\ell}}{\partial y^{2}}+g \frac{\rho_{\ell}-\rho_{\mathrm{v}}}{\rho_{\ell}} \cos (\phi) \\
& u_{\ell} \frac{\partial T_{\ell}}{\partial x}+v_{\ell} \frac{\partial T_{\ell}}{\partial y}=\alpha_{\ell} \frac{\partial^{2} T_{\ell}}{\partial y^{2}}
\end{aligned}
$$




\subsubsection{Conditions aux limites}

Aux équations (1)-(6), nous associons les conditions aux limites suivantes :

$-y=0$, la paroi est isotherme et la vitesse obéit à la condition de non glissement :

$$
u_{\mathrm{p}}=v_{\mathrm{p}}=0, \quad T_{\mathrm{p}}=T_{\mathrm{w}}
$$

- À l'interface couche poreuse/liquide pur $y=H$, la continuité des vitesses, des températures, des contraintes de cisaillement et des densités de flux de chaleur conduit aux relations ci-dessous :

$$
\begin{aligned}
& u_{\mathrm{p}}=u_{\ell}, \quad T_{\mathrm{p}}=T_{\ell} \\
& \left.\mu_{\mathrm{eff}} \frac{\partial u_{\mathrm{p}}}{\partial y}\right|_{H}=\left.\mu_{\ell} \frac{\partial u_{\ell}}{\partial y}\right|_{H} \\
& \left.k_{\mathrm{eff}} \frac{\partial T_{\mathrm{p}}}{\partial y}\right|_{H}=\left.k_{\ell} \frac{\partial T_{\ell}}{\partial y}\right|_{H}
\end{aligned}
$$

- À la frontière liquide-vapeur $y=\delta$,

$$
\left.\frac{\partial u_{\ell}}{\partial y}\right|_{y=\delta}=0, \quad T_{\ell}=T_{\mathrm{s}}
$$

Le bilan thermique est donné par :

$$
\left.k_{\mathrm{eff}} \frac{\partial T}{\partial y}\right|_{y=0}=\frac{\mathrm{d}}{\mathrm{d} x}\left\{\int_{0}^{\delta} \rho_{L}\left[h_{f g}+c_{\mathrm{p}}\left(T_{\mathrm{s}}-T_{\xi}\right)\right] u_{\xi} \mathrm{d} y\right\}
$$

où $\xi=p$ ou $\ell$.

\subsection{Transformation des équations}

Les équations de transferts (1)-(6), les conditions aux limites qui leurs sont associées $(7 \mathrm{a}-\mathrm{i})$ et le bilan thermique (8) sont adimensionnalisés en utilisant les variables sans dimensions suivantes [9] :

$$
\begin{aligned}
& y^{*}=\frac{y}{\sqrt{K}} \quad x^{*}=\frac{x}{\sqrt{K}} \quad H^{*}=\frac{H}{\sqrt{K}} \quad \delta^{*}=\frac{\delta}{\sqrt{K}} \\
& u^{*}=\frac{u_{\xi}}{u_{r}} \quad \theta_{\xi}=\frac{T_{\xi}-T_{\mathrm{w}}}{T_{\mathrm{s}}-T_{\mathrm{w}}}
\end{aligned}
$$

où $u_{r}=\frac{K}{\nu_{\text {eff }}} g \cos (\phi)$.

Le plan $\left(x^{*}, y^{*}\right)$ est ramené au plan $(X, \eta)$ à l'aide des transformations suivantes [16] :

- Dans la couche poreuse, $0 \leq y^{*} \leq H^{*}$ :

$$
X=x^{*}, \quad \eta=y^{*} / H^{*}
$$

- Dans le liquide pur, $H^{*} \leq y^{*} \leq \delta^{*}$ :

$$
X=x^{*}, \quad \eta=1+\left(y^{*}-H^{*}\right) /\left(\delta^{*}-H^{*}\right)
$$

Dans les deux milieux, les équations adimensionnelles et les conditions aux limites qui leur sont associées s'écrivent comme dans les sections suivantes.

\subsubsection{Couche poreuse : $0<\eta<1$}

$$
\begin{aligned}
& \frac{\partial u_{\mathrm{p}}^{*}}{\partial X}+\frac{1}{H^{*}} \frac{\partial v_{\mathrm{p}}^{*}}{\partial \eta}=0 \\
& \frac{1}{H^{* 2}} \frac{\partial^{2} u_{\mathrm{p}}^{*}}{\partial \eta^{2}}-u_{\mathrm{p}}^{*}+1=0 \\
& \frac{\partial^{2} \theta_{\mathrm{p}}}{\partial \eta^{2}}=0
\end{aligned}
$$

2.3.2 Liquide pur : $1<\eta<2$

$$
\left(\delta^{*}-H^{*}\right) \frac{\partial u_{\ell}^{*}}{\partial X}-(\eta-1) \frac{\mathrm{d} \delta^{*}}{\mathrm{~d} X} \frac{\partial u_{\ell}^{*}}{\partial \eta}+\frac{\partial v_{\ell}^{*}}{\partial \eta}=0
$$

$$
\begin{array}{r}
u_{\ell}^{*}\left[\frac{\partial u_{\ell}^{*}}{\partial X}-\frac{\eta-1}{\delta^{*}-H^{*}} \frac{\mathrm{d} \delta^{*}}{\mathrm{~d} X} \frac{\partial u_{\ell}^{*}}{\partial \eta}\right]+\frac{v_{\ell}^{*}}{\delta^{*}-H^{*}} \frac{\partial u_{\ell}^{*}}{\partial \eta}= \\
\frac{1}{R e_{K}}\left[1+\frac{1}{\left(\delta^{*}-H^{*}\right)^{2}} \frac{\partial^{2} u_{\ell}^{*}}{\partial \eta^{*^{2}}}\right] \\
u_{\ell}^{*}\left[\frac{\partial \theta_{\ell}}{\partial X}-\frac{\eta-1}{\delta^{*}-H^{*}} \frac{\mathrm{d} \delta^{*}}{\mathrm{~d} X} \frac{\partial \theta_{\ell}}{\partial \eta}\right]+\frac{v_{\ell}^{*}}{\delta^{*}-H^{*}} \frac{\partial \theta_{\ell}}{\partial \eta}= \\
\frac{1}{R e_{K} \operatorname{Pr}\left(\delta^{*}-H^{*}\right)^{2}} \frac{\partial^{2} \theta_{\ell}}{\partial \eta^{2}}
\end{array}
$$

\subsubsection{Conditions aux limites}

- À la paroi, $\eta=0$ :

$$
u_{\mathrm{p}}^{*}=v_{\mathrm{p}}^{*}=0, \quad \theta_{\mathrm{p}}=0
$$

- À l'interface couche poreuse/liquide pur, $\eta=1$ :

$$
\begin{aligned}
& u_{\mathrm{p}}^{*}=u_{\ell}^{*} \quad \theta_{\mathrm{p}}=\theta_{\ell} \\
& \frac{\mu^{*}}{H^{*}} \frac{\partial u_{p}^{*}}{\partial \eta}\left|=\frac{1}{\left(\delta^{*}-H^{*}\right)} \frac{\partial u_{\ell}^{*}}{\partial \eta}\right| \\
& \frac{1}{H^{*}} \frac{\partial \theta_{p}}{\partial \eta}\left|=\frac{k^{*}}{\left(\delta^{*}-H^{*}\right)} \frac{\partial \theta_{\ell}}{\partial \eta}\right|
\end{aligned}
$$

- À l'interface liquide/vapeur, $\eta=2$ :

$$
\frac{\partial u_{\ell}^{*}}{\partial \eta}=0 \quad \theta_{\ell}=1
$$


L'expression (8) s'écrit :

$$
\begin{aligned}
\left.\frac{J a}{(P e)_{\mathrm{eff}}} \frac{1}{H^{*}} \frac{\partial \theta_{p}}{\partial \eta}\right|_{\eta=0}= \\
\frac{\mathrm{d}}{\mathrm{d} x^{*}}\left[H^{*} \int_{0}^{1}\left\{1+J a\left(1-\theta_{p}\right)\right\} u_{\mathrm{p}}^{*} \mathrm{~d} \eta\right] \\
+\frac{\mathrm{d}}{\mathrm{d} x^{*}}\left[\int_{1}^{2}\left(\delta^{*}-H^{*}\right)\left\{1+J a\left(1-\theta_{\ell}\right)\right\} u_{\ell}^{*} \mathrm{~d} \eta\right]
\end{aligned}
$$

avec :

$(P e)_{\mathrm{eff}}=k^{*} \operatorname{Pr} \operatorname{Re}_{K}:$ nombre de Peclet effectif.

$\operatorname{Pr}=\frac{\mu_{\ell} C p_{\ell}}{k_{\ell}}:$ nombre de Prandtl.

$R e_{K}=\frac{u_{r} \sqrt{K}}{\nu_{\text {eff }}}:$ nombre de Reynolds basé sur $\sqrt{K}$.

$k^{*}=\frac{k_{\ell}}{k_{\mathrm{eff}}}:$ conductivité thermique adimensionnelle.

$\mu^{*}=\frac{\mu_{\mathrm{eff}}}{\mu_{\ell}}$ : viscosité dynamique adimensionnelle.

$J a=\frac{C p_{\ell}\left(T_{\mathrm{S}}-T_{\mathrm{w}}\right)}{h_{f g}}:$ nombre de Jacob.

\section{Méthodes de résolution}

\subsection{Solution analytique}

En tenant compte des conditions aux limites (19a-h) et en négligeant les termes d'inertie et de la convection d'enthalpie dans le liquide pur, les profils de vitesse et de température s'expriment respectivement dans les deux domaines par :

$u_{\mathrm{p}}^{*}(\eta)=\left[S h\left(H^{*}\right)+\left(\delta^{*}-H^{*}\right)\right] \frac{S h\left(\eta H^{*}\right)}{C h\left(H^{*}\right)}+1-C h\left(\eta H^{*}\right)$

$\theta_{\mathrm{p}}(\eta)=\frac{H^{*} k^{*}}{H^{*}\left(k^{*}-1\right)+\delta^{*}} \eta$

$u_{\ell}^{*}(\eta)=a_{2} \eta^{2}+a_{1} \eta+a_{0}$

$\theta_{\ell}(\eta)=\frac{\left(\delta^{*}-H^{*}\right)}{\delta^{*}+H^{*}\left(k^{*}-1\right)}(\eta-1)+\frac{k^{*} H^{*}}{\delta^{*}+H^{*}\left(k^{*}-1\right)}$

où

$$
\begin{aligned}
a_{2}=-\frac{1}{2} \mu^{*}\left(\delta^{*}-H^{*}\right)^{2}, \quad a_{1}=2 \mu^{*}\left(\delta^{*}-H^{*}\right)^{2} \\
a_{0}=u_{i}^{*}-\frac{3}{2} \mu^{*}\left(\delta^{*}-H^{*}\right)^{2}
\end{aligned}
$$

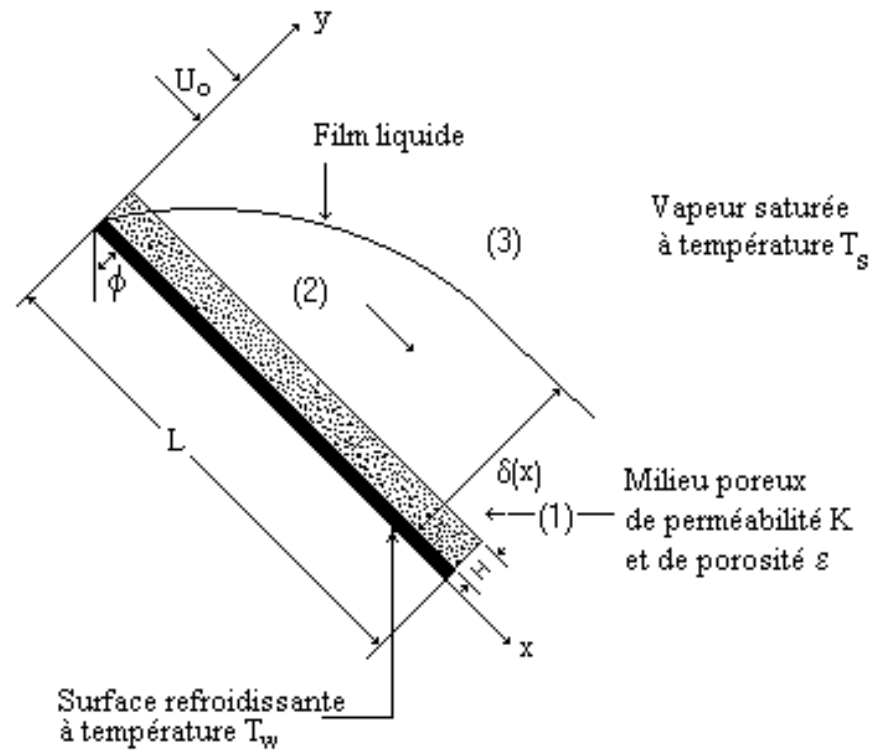

Fig. 1. Modèle physique et système de coordonnées.

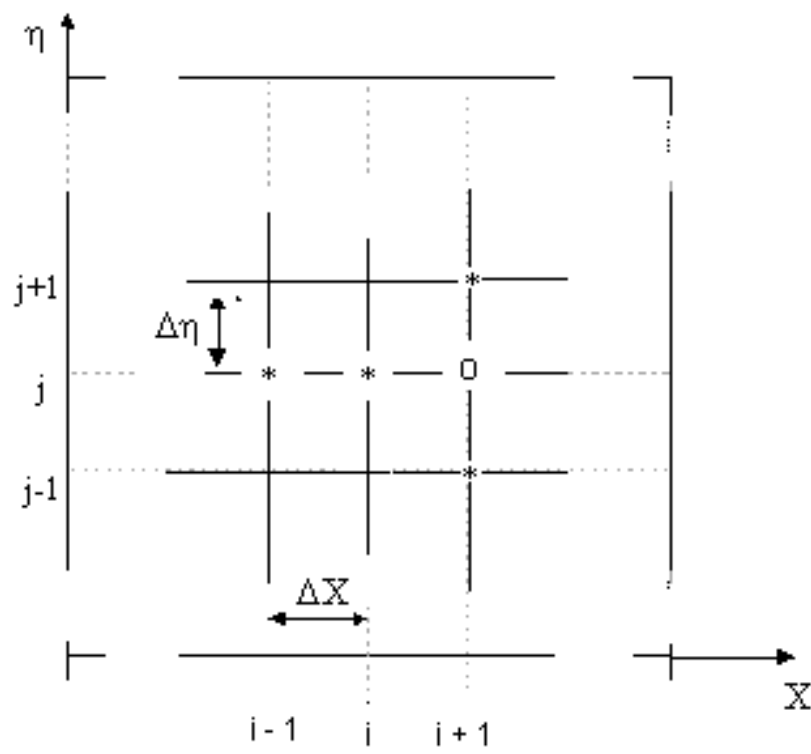

Fig. 2. Maillage. * Nouds intervenant dans la discrétisation.

- Noud sur lequel porte le calcul.

\subsection{Méthode numérique}

Les équations (13)-(18) associées aux conditions aux limites $(19 \mathrm{a}-\mathrm{h})$ ont été résolues à l'aide d'une méthode aux différences finies implicite [16]. Dans ce type de schéma les dérivées partielles sont exprimées aux nœuds d'indices $(i+1, j)$ du maillage $\left(X_{i}, \eta_{j}\right)$ (Fig. 2) :

$$
\Delta X=X_{i+1}-X_{i}, \quad \Delta \eta=\eta_{j}-\eta_{j-1}
$$

$(i, j)$ et $(\Delta X, \Delta \eta)$ représentent respectivement les nœuds et les pas suivant $X$ et $\eta$. Les expressions des dérivées partielles intervenant dans les équations traitées sont déduites à partir du développement de Taylor. Les termes 
non linéaires de ces équations sont examinés à l'aide des approximations proposées dans la référence [17]. En négligeant les termes d'ordre $(m+1)$ de la fonction $F\left(u_{\mathrm{p}}^{*}, \theta_{\mathrm{p}}\right.$ ou $\left.\delta^{*}\right)$, l'erreur commise est inférieure à $\frac{|e|^{m+1}}{(m+1) !}\left[F^{(m+1)}\right]_{\max },(e=\Delta X$ ou $\Delta \eta, m=1$ ou 2$)[18]$.

Le maillage comporte NX stations d'indice $i$. En chaque station, $j$ varie de 2 à $(\mathrm{NT}-1)$, puisque à la paroi et à la frontière du film liquide, l'écoulement est imposé par les conditions aux limites. Dans les deux domaines, les équations du mouvement discrétisées peuvent s'écrire sous la forme suivante à chaque pas $i$ :

$$
[\mathrm{B}] \cdot\left[\mathrm{U}^{i+1}\right]=[\mathrm{D}]
$$

où $[\mathrm{B}]$ est une matrice tridiagonale, $\left[\mathrm{U}^{i+1}\right]$ est un vecteur vitesse (inconnu) et $[\mathrm{D}]$ est un vecteur colonne (deuxième membre).

On est donc amené à résoudre un système d'équations linéaires dont les inconnues sont les $\left[u_{\xi}^{*}\right]_{j}^{i+1}$ à l'aide de la méthode de Gauss. Un raisonnement similaire est adopté pour déterminer les températures. La composante normale de la vitesse est obtenue à partir de l'équation de continuité discrétisée. Pour résoudre le système linéaire, on calcule $u_{\xi}^{*}$ et $\theta_{\xi}$ pour des valeurs arbitraires de l'épaisseur du film de condensat $\delta^{*}$ et on évalue l'erreur sur le bilan thermique afin de corriger ces valeurs. Une nouvelle valeur de $\delta^{*}$ est calculée si nécessaire par la méthode de la sécante. La convergence est obtenue après quelques itérations pour une erreur relative sur $\delta^{*}$ de $10^{-6}$. Pour démarrer les calculs, les profils des vitesses et des températures sont indispensables pour les deux premiers plans de calcul $(i=1$ et $i=2)$. Ils sont obtenus en s'appuyant sur les solutions analytiques ci-dessus (Éqs. (21) à (24)).

\section{Résultats}

Notre étude permet d'examiner et de mettre en évidence le rôle des différents paramètres influents tels que : l'inclinaison $\phi$, le nombre de Reynolds $R e_{K}$, l'épaisseur adimensionnelle de la couche poreuse $H^{*}$ et la conductivité thermique adimensionnelle $k^{*}$, sur le comportement hydrodynamique et thermique du film liquide. Les calculs ont été effectués pour les valeurs suivantes :

$-\phi=0^{\circ}, 30^{\circ}$ et $60^{\circ} ; R e_{K}=2,5$ et $10 ; H^{*}=0,254$; 0,1 et 0,0254 .

$-k^{*}=3,3 \times 10^{-3} ; 0,5$ et 1,$0 ; \mathrm{Ja}=10^{-2} ; \mathrm{Pr}=2$, $K=10^{-8} \mathrm{~m}^{2}$ et $\mu^{*}=1$.

Compte tenu de l'objectif de cette étude, la présentation des profils de vitesse et de température est limitée à la couche poreuse.

\section{1 Épaisseur adimensionnelle du film liquide}

Sur les figures $3 \mathrm{a}-\mathrm{d}$, nous présentons l'évolution de l'épaisseur adimensionnelle du film liquide $\delta^{*}$ en fonction de la position adimensionnelle $X$ le long de la plaque poreuse. La première parmi elles (Fig. 3a), correspond aux différentes angles d'inclinaison $\left(\phi=0^{\circ}, 30^{\circ}\right.$ et $\left.60^{\circ}\right)$. L'épaisseur du film liquide est une fonction croissante de l'abscisse et augmente avec l'inclinaison $\phi$ de la plaque. Cette évolution de l'épaisseur provoque des variations des échanges thermiques entre la paroi et la phase vapeur (voir Fig. 6a) qui sont discutés à la section 4.3. Elle résulte de l'influence de la gravité sur les forces volumiques d'origines thermique qui ont tendance à disparaître lorsque l'angle d'inclinaison tend vers $90^{\circ}$ (cas d'une plaque horizontale). On remarque que l'inertie du liquide pur provoque une diminution de l'épaisseur du film en comparaison à celle obtenue en l'absence d'inertie.

Les figures $3 \mathrm{~b}-\mathrm{d}$ montrent respectivement l'influence du nombre de Reynolds $R e_{K}$ basé sur la racine de la conductivité hydraulique $\sqrt{K}$ (effet de la diffusivité cinématique effective), de l'épaisseur de la couche poreuse $H^{*}$ (effet de la forme de la couche poreuse) et de la conductivité thermique adimensionnelle $k^{*}$ (effet de la conductivité thermique effective), sur l'évolution de l'épaisseur adimensionnelle $\delta^{*}$ du film de condensat le long d'une plaque plane verticale $\left(\phi=0^{\circ}\right)$. La figure $3 \mathrm{~b}$, témoigne d'une nette augmentation de $\delta^{*}$ lorsque le nombre de Reynolds $R e_{K}$ diminue (la diffusivité cinématique effective augmente). Cette évolution montre que la vitesse de l'écoulement du film de condensat et sa diffusivité jouent un rôle essentiel sur le comportement du film et sur les transferts entre le milieu poreux et ce film. Nous notons que l'épaisseur du film de condensat est d'autant plus élevée que la diffusivité est importante ce qui montre que la présence d'un film de condensat ruisselant sur un matériau poreux s'accompagne d'une augmentation de l'épaisseur de ce film d'autant plus importante que la viscosité du fluide est élevée. Le même comportement du film est observé en en diminuant la conductivité thermique adimensionnelle $k^{*}$ (Fig. 3d). Quant à la figure 3c, elle montre une légère augmentation du film provoquée par un acroissement de $H^{*}$. Ceci peut être interprété par le fait que la couche poreuse permet d'avoir un meilleur échange thermique entre la paroi et la phase vapeur.

Sur ces figures, nous avons procédé à la mise en évidence de l'influence du terme d'inertie relatif au liquide pur, sur l'épaississement du film dans le cas d'une plaque plane verticale. Nous observons que l'absence de ce terme provoque une augmentation de l'épaisseur du film.

\subsection{Profils de vitesse et de température}

À la position $X=313$ sur la plaque poreuse, les variations des profils de vitesse et de température dans le film de condensat sont respectivement montrées sur les figures $4 \mathrm{a}-\mathrm{d}$ et $5 \mathrm{a}-\mathrm{d}$, en fonction de l'ordonnée $\eta$ pour les paramètres influents mentionnés précédemment $\left(\phi, R e_{K}\right.$, $H^{*}$ et $\left.k^{*}\right)$.

L'influence de la gravité sur la vitesse longitudinale, est d'autant plus significative que l'angle d'inclinaison de la plaque par rapport à la verticale est faible (Fig. 4a). En effet, au voisinage d'une plaque les forces de flottabilités 



Fig. 3. Variations de l'épaisseur du film de condensat au long de la plaque poreuse. AT : avec terme d'inertie, ST : sans terme d'inertie. (a) Effet de l'inclinaison $\phi: R e_{K}=5 ; k^{*}=3,3 \times 10^{-3} ; H^{*}=0,254$. (b) Effet du nombre de Reynolds $R e_{K}: \phi=0^{\circ}$; $k^{*}=3,3 \times 10^{-3} ; H^{*}=0,254$. (c) Effet de l'épaisseur de la couche poreuse $H^{*} . \phi=0^{\circ} ; k^{*}=3,3 \times 10^{-3} ; R e_{K}=5$. (d) Effet de la conductivité thermique effective adimensionnelle $k^{*} . \phi=0^{\circ} ; \operatorname{Re}_{K}=5 ; H^{*}=0,254$.

sont d'autant plus importantes que l'angle d'inclinaison par rapport à la verticale est faible. Ainsi, l'écoulement devient plus intense lorsque l'angle d'inclinaison de la plaque par rapport à la verticale passe de $60^{\circ}$ à $0^{\circ}$. Les figures $4 \mathrm{~b}-\mathrm{d}$ confirment le rôle des paramètres $R e_{K}, H^{*}$ et $k^{*}$ reportés ci-dessus (Figs. 3c, d) sur le comportement hydrodynamique de l'écoulement. Ce dernier peut être accéléré en diminuant le nombre de Reynolds (Fig. 4b) ou en augmentant l'épaisseur de la couche poreuse (Fig. 4c). Les variations de la conductivité thermique adimensionnelles s'accompagnent d'une faible variation de la composante longitudinale de la vitesse (Fig. 4d). Cela découle $\mathrm{du}$ fait que les termes de convection d'enthalpie sont négligeables dans la couche poreuse. La comparaison entre les valeurs de la vitesse longitudinale déduites de la relation (21) avec celles obtenues en résolvant numériquement l'équation (14) montre un bon accord qualitatif et quantitatif (écart <10\%).

Les figures $5 \mathrm{a}-\mathrm{d}$ précisent une évaluation des champs de température dans le substrat poreux. Ces profils sont linéaires dans tous les cas exposés. Cette linéarité est due au fait que les termes convectifs sont négligeables.
Sur les figures 5-c, les valeurs de la température sont généralement faibles par comparaison à celles de la figure 5d. Les gradients de température sont moins sensibles aux variations des paramètres $\phi$ (Fig. 5a) et $R e_{K}$ (Fig. 5b) par comparaison aux grandeurs adimensionnelles $H^{*}$ (Fig. 5c) et de $k^{*}$ (Fig. 5d). Nous précisons pour cette dernière représentation (Fig. 5d) que la conductivité thermique effective du milieu poreux permet de quantifier les transferts conductifs au sein du milieu. Finalement, sur chacune des figures relatives aux profils de température, une comparaison entre les valeurs des températures déduites de l'expression analytique (22) et celles obtenues en résolvant numériquement l'équation (15) montre un écart maximale de l'ordre de $12 \%$.

\subsection{Transferts thermiques}

L'analyse de l'influence des différents paramètres précités sur les transferts, qui régissent la condensation en film sur une plaque poreuse, nécessite la détermination 

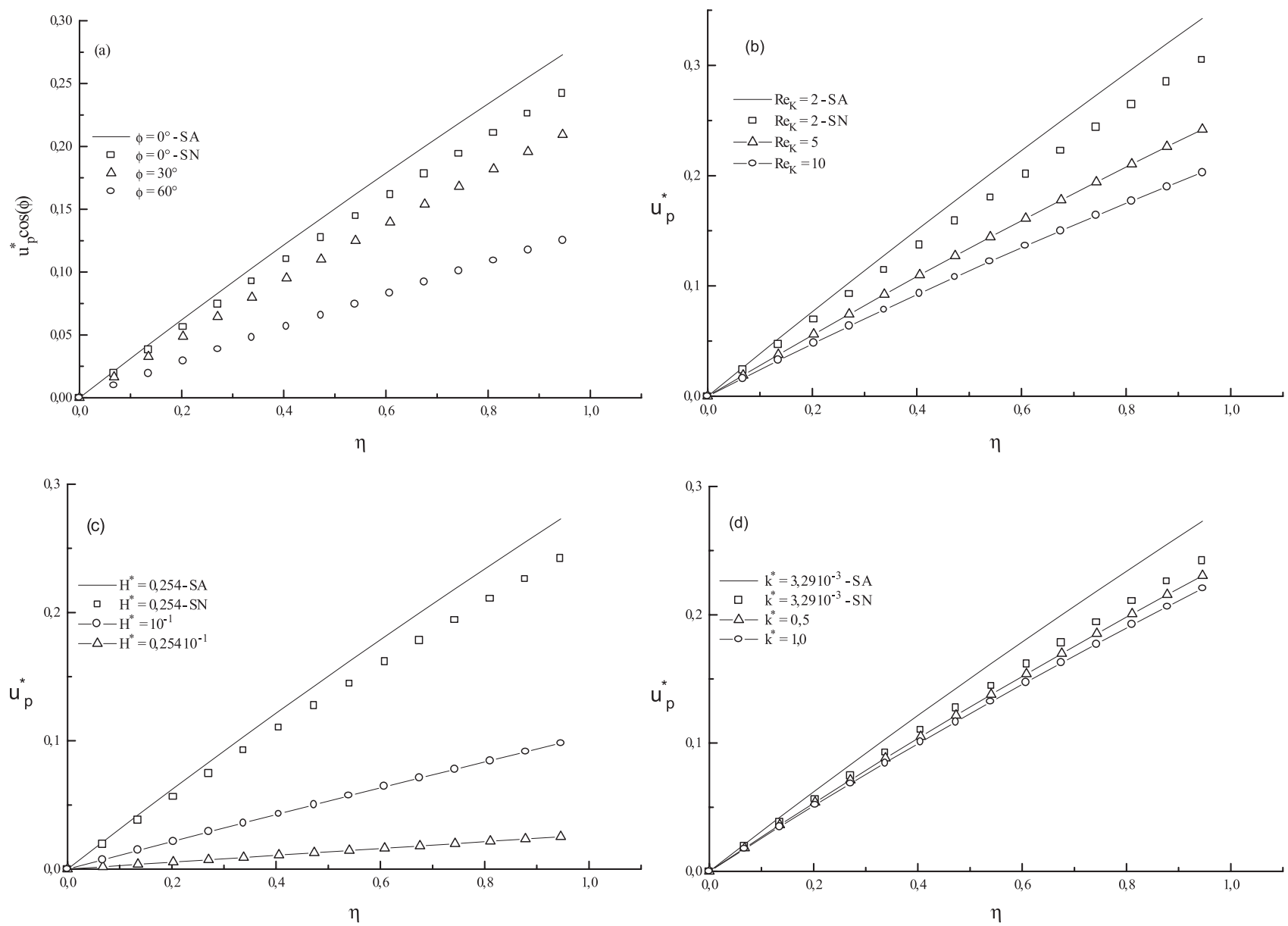

Fig. 4. Profils de vitesse dans le substrat poreux, à la position $X=313$ sur la plaque. SA : solution analytique (Éq. (21)), SN : solution numérique. (a) Effet de l'inclinaison $\phi: R e_{K}=5 ; k^{*}=3,3 \times 10^{-3} ; H^{*}=0,254$. (b) Effet du nombre de Reynolds $R e_{K}: \phi=0^{\circ} ; k^{*}=3,3 \times 10^{-3} ; H^{*}=0,254$. (c) Effet de l'épaisseur de la couche poreuse $H^{*} \cdot \phi=0^{\circ} ; k^{*}=3,3 \times 10^{-3}$; $R e_{K}=5$. (d) Effet de la conductivité thermique effective adimensionnelle $k^{*} . \phi=0^{\circ} ; R e_{K}=5 ; H^{*}=0,254$.

des nombres de Nusselt local $N u_{x}$ et moyen $N u_{L}$, définis respectivement par :

$$
\begin{aligned}
& \left.N u_{x}=\frac{1}{k^{*} H^{*}} X \frac{\partial \theta_{p}}{\partial \eta}\right]_{0} \\
& N u_{L}=\frac{1}{L} \int_{0}^{L} N u_{x} \mathrm{~d} x
\end{aligned}
$$

L'expression (25) montre que le nombre de Nusselt local dépend essentiellement du gradient de température à la paroi. Au paragraphe précédent nous avons indiqué que les variations de ce gradient dépendent du nombre de Reynolds, de l'angle d'inclinaison de la plaque, de l'épaisseur de la couche poreuse et de sa conductivité thermique. L'analyse des figures $6 \mathrm{a}-\mathrm{d}$ confirme les résultats précédents.

Afin de valider nos résultats, nous avons eu recours aux résultats expérimentaux de Renken et al. [1]. Dans un premier temps, nous avons procédé à une confrontation entre les valeurs du nombre de Nusselt moyen déduit de notre modèle avec celles de la référence [1], pour plusieurs différences de température $\Delta T=\left(T_{\mathrm{S}}-T_{\mathrm{w}}\right)$ entre la paroi et la vapeur (Fig. 7). Cette comparaison est effectuée pour une plaque verticale $\left(\phi=0^{\circ}\right)$ et pour trois valeurs du nombre de Reynolds $\operatorname{Re}_{K}\left(R e_{K}=2\right.$, 5 et 10). À partir de cette dernière figure, il est évident que les prévisions de nos calculs sont en accord relatif avec les résultats expérimentaux de Renken et al. [1]. L'écart observé entre nos résultats et ceux expérimentaux de Renken et al. [1] est d'autant plus important que la différence de température entre la vapeur et la paroi est relativement faible, ce qui correspond aux petites épaisseurs du film liquide. Cet écart peut être expliqué par le fait que dans notre modèle, nous avons supposé l'absence des gaz non condensables (vapeur pure) et que la vapeur et le condensat sont séparés par une frontière distincte.

D'autre part, la figure 8 montre clairement l'effet de la gravité sur les transferts thermiques en représentant le nombre de Nusselt moyen $N u_{L}$ en fonction de l'inclinaison. Cette représentation permet de conclure que le degré de perfectionnement des échanges thermiques continue à augmenter au fur et à mesure que l'inclinaison diminue [la force de gravite augmente lorsque $\cos (\phi)$ augmente]. 

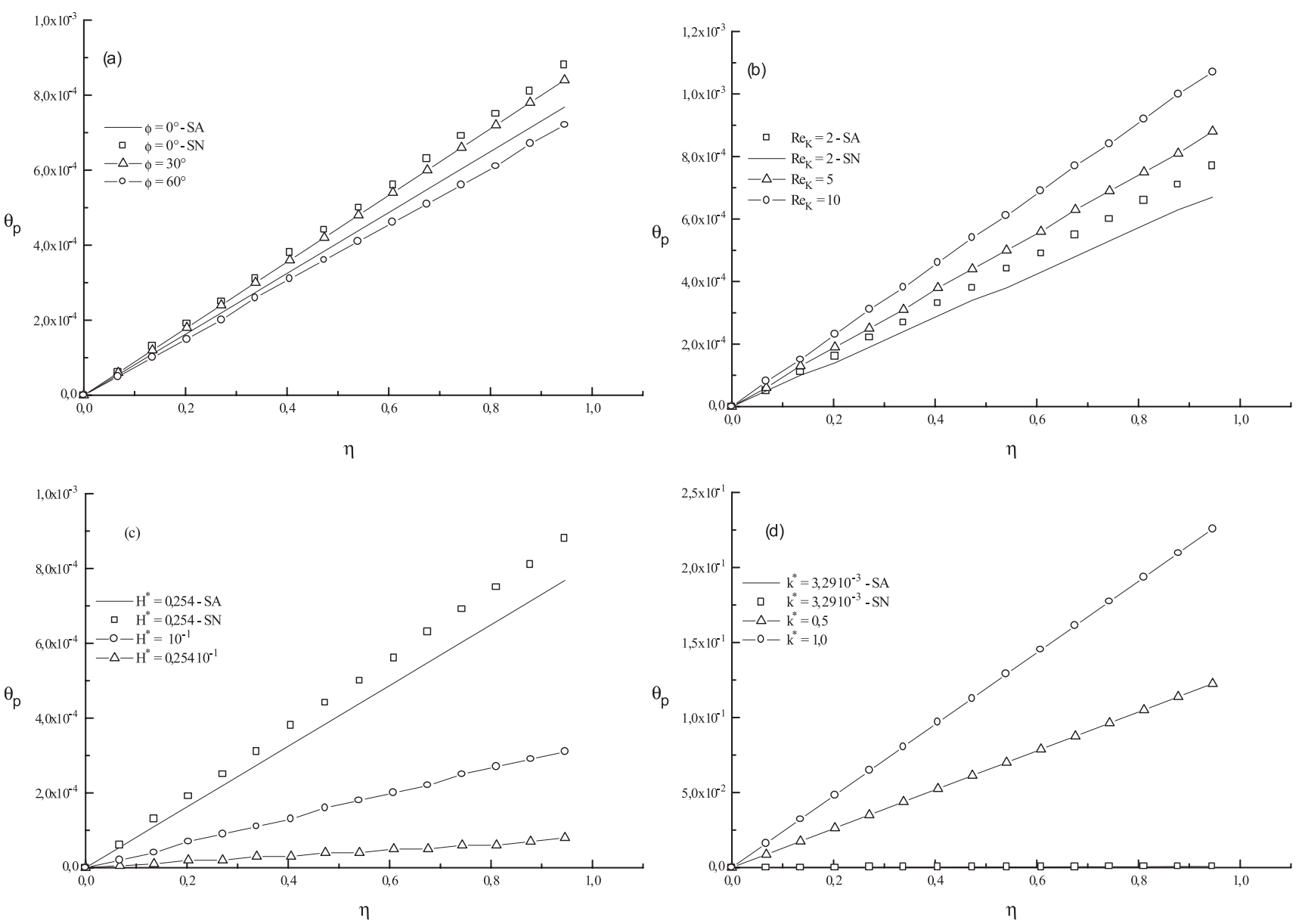

Fig. 5. Profils de température dans le substrat poreux, à la position $X=313$ sur la plaque. SA : solution analytique (Éq. (22)), SN : solution numérique. (a) Effet de l'inclinaison $\phi: R e_{K}=5 ; k^{*}=3,3 \times 10^{-3} ; H^{*}=0,254$. (b) Effet du nombre de Reynolds $R e_{K}: \phi=0^{\circ} ; k^{*}=3,3 \times 10^{-3} ; H^{*}=0,254$. (c) Effet de l'épaisseur de la couche poreuse $H^{*} . \phi=0^{\circ} ; k^{*}=3,3 \times 10^{-3} ;$ $R e_{K}=5$. (d) Effet de la conductivité thermique effective adimensionnelle $k^{*} . \phi=0^{\circ} ; R e_{K}=5 ; H^{*}=0,254$.

Pour trois épaisseurs de la couche poreuse, les nombres de Nusselt moyens $\left(N u_{L}\right)$ déduits de notre modèle, sont comparés à ceux obtenus à partir des mesures reportées dans la référence [1]. Dans cette figure, l'effet de l'épaisseur de la couche poreuse est de nouveau prononcé. Sachant que les résultats expérimentaux de la figure 8 ont été effectués à une différence de température $\Delta T=35 \mathrm{~K}$, l'écart observé entre nos résultats et ceux de la référence [1] a été justifié en interprétant la figure 7. La comparaison montre un accord quantitatif acceptable.

\section{Conclusion}

En utilisant le modèle de Darcy-Brinkman pour décrire le mouvement dans la couche poreuse et en tenant compte des termes d'inertie et de convection d'enthalpie dans le liquide pur, nous avons analysé analytiquement et numériquement les transferts de chaleur dans un film de condensat s'écoulant sur une plaque inclinée par rapport à la verticale, couverte par un substrat poreux.

Nous avons analysé l'influence de :

- l'inclinaison de la plaque et des termes d'inertie,
- l'épaisseur de la couche poreuse,

- la diffusivité cinématique effective (influence du nombre de Reynolds $R_{K}$ ),

- la conductivité thermique adimensionnelle, sur les échanges thermiques entre la paroi et la vapeur.

Nos résultats validés par une comparaison avec les données expérimentales de Renken et al. [1] montrent en particulier que :

- L'utilisation d'une paroi recouverte d'une couche poreuse améliore les transferts thermiques.

- L'absence du terme d'inertie relatif au liquide pur, provoque une augmentation de l'épaisseur du film de condensat.

- Les échanges thermiques locaux augmentent en diminuant l'angle d'inclinaison par rapport à la verticale.

- La diffusivité cinématique effective joue un rôle essentiel sur le comportement du film et sur les transferts thermiques entre la paroi et la phase vapeur.

- Dans la couche poreuse, les gradients de température sont moins sensibles aux variations de l'angle d'inclinaison et au nombre de Reynolds qu'à l'épaisseur et la conductivité thermique du milieu poreux. 

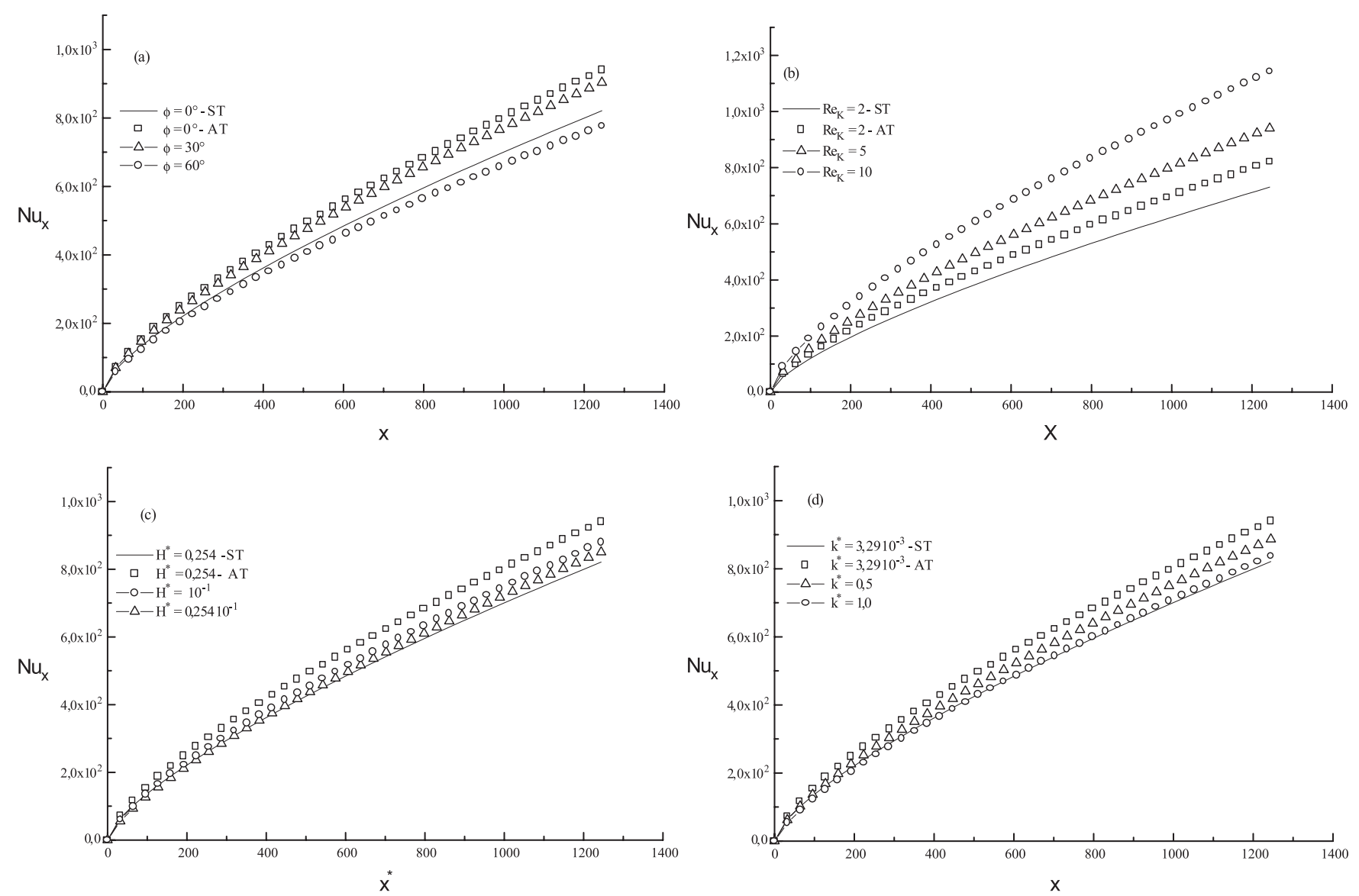

Fig. 6. Évolution du nombre de Nusselt local en fonction de la position sur la plaque poreuse. AT : avec terme d'inertie, ST : sans terme d'inertie. (a) Effet de l'inclinaison $\phi: R e_{K}=5 ; k^{*}=3,3 \times 10^{-3} ; H^{*}=0,254$. (b) Effet du nombre de Reynolds $R e_{K}: \phi=0^{\circ} ; k^{*}=3,3 \times 10^{-3} ; H^{*}=0,254$. (c) Effet de l'épaisseur de la couche poreuse $H^{*} . \phi=0^{\circ} ; k^{*}=3,3 \times 10^{-3}$; $R e_{K}=5$. (d) Effet de la conductivité thermique effective adimensionnelle $k^{*} . \phi=0^{\circ} ; R e_{K}=5 ; H^{*}=0,254$.

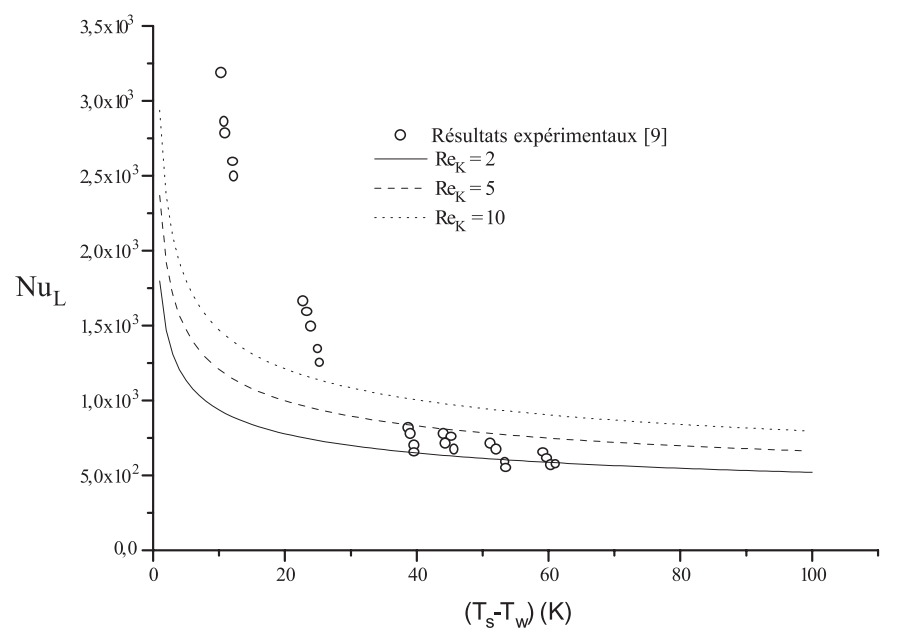

Fig. 7. Évolution du nombre de Nusselt moyen en fonction de $\left(T_{\mathrm{S}}-T_{\mathrm{w}}\right)$. Comparaison avec les résultats expérimetaux de Renken et al. [1].

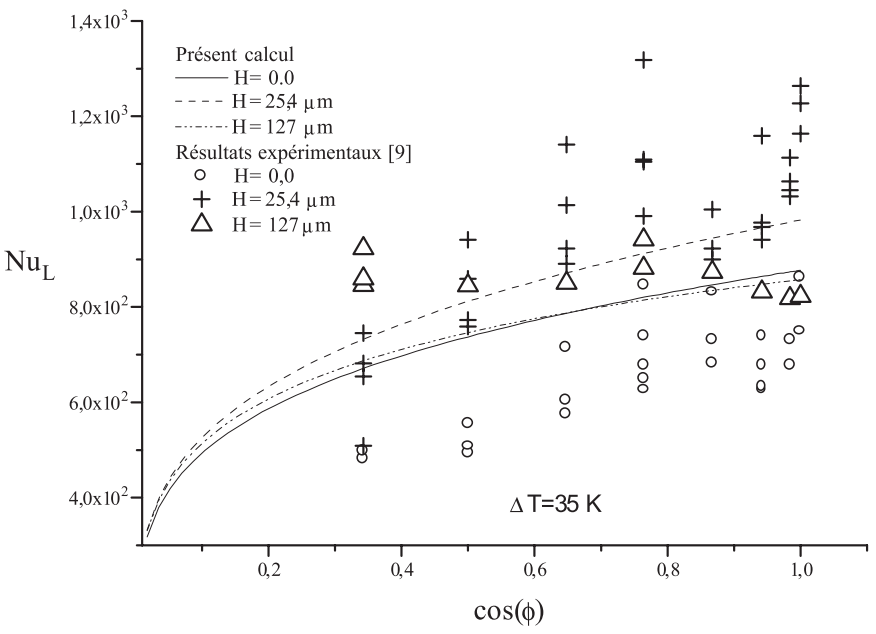

Fig. 8. Évolution du nombre de Nusselt moyen en fonction de l'angle $\phi$. Comparaison avec les résultats expérimetaux de Renken et al. [1]. 


\section{Références}

[1] K.-J. Renken, M. Aboye, Experiments on film condensation promotion within thin inclined porous coatings, Int. J. Heat Mass Transf. 36 (1993) 1347-1355

[2] P. Cheng, A. Verma, The effect of subcooled liquid on film boiling about a vertical heated surface in a porous medium, Int. J. Heat Mass Trans. 24 (1981) $1151-1160$

[3] C.-T. Avedisian, J. Koplic, Liedenfrost boiling of methanol droplets on hot porous/ceramic surfaces, Int. J. Heat Mass Trans. 30 (1982) 379-393

[4] N.-M. Afgan, L.-A. Jovic, S.-A. Kovalev, V.-A. Lenykov, Boiling heat transfer from surfaces with porous layers, Int. J. Heat Mass Trans. 28 (1986) 91-94

[5] S.-V. Konev, Mitrovic J., An explanation for the augmentation of heat transfer during boiling in capillary structures, Int. J. Heat Mass Trans. 29 (1981) 1151-1160

[6] D.-W. Woodruff, J.-W. Westwater, Steam condensation on various gold surface, J. Heat Trans. 103 (1981) 685-692

[7] Q. Zhao, D. Zhang, X. Zhu, D. Xu, Z. Lin, J. Lin, Industrial application of dropwise condensation, Proceeding of the Ninth International Heat Transfer Conference, Hemisphere Washington, DC, 4, 1990, pp. 391-394

[8] A. Shekarriz, O.-A. Plumb, Enhancement of film condensation using porous fins, J. Thermophysics and Heat Trans. 3(3) (1989) 309-314

[9] K.-J. Renken, D.-J. Soltykiewicz, D. Poulikakos, A study of laminar film condensation on a vertical surface with a porous coating, Int. Comm. Heat Mass Transf. 16 (1989) 181-192
[10] K.-J. Renken, C.-D. Mueller, Measurements of enhanced film condensation utilizing a porous metallic coating, J. Thermophys. Heat Transf. 7 (1993) 148-152

[11] K.-J. Renken, M. Aboye, Analysis of film condensation within inclined thin porous-layer coated surfaces, Int. J. Heat and Fluid Flow 14 (1993) 48-53

[12] K.-J. Renken, M.-R. Raich, Forced convection steam condensation experiments within thin porous coatings, Int. J. Heat Mass Transf. 39 (1996) 2937-2945

[13] R. Chaynane, M. Asbik, H. Boushaba, A. Khmou, Étude de la condensation en film laminaire d'une vapeur pure et saturée à la paroi d'une plaque poreuse inclinée, Actes du 5 Congrès de Mécanique, Meknès, Maroc, 2001, Tome 2, pp. $151-152$

[14] M. Asbik, R. Chaynane, B. Zeghmati, J. Bresson, Étude analytique de la condensation en film laminaire en convection forcée d'une vapeur pure et saturée sur une paroi poreuse verticale : Effet de la dispersion thermique, Actes du Congrès SFT 2002, Vittel, 3-6 juin 2002, pp. 381-386

[15] M. Asbik, R. Chaynane, H. Boushaba, B. Zeghmati, A. Khmou, Analytical investigation of forced convection film condensation on a vertical porous-layer coated surface, J. Heat Trans. 40 (2003) 143-155

[16] M. Asbik, D. Ouldhadda, Z. Zeghmati, A. Khmou, A. Il Idrissi, Forced Convection laminar film condensation of downward flowing vapor on a single horizontal elliptic cylinder or a bank of elliptical tubes, Numerical Heat Transfer Part A, 37(5) (2000) 511-544

[17] A.A. Dale, J.C. Tannehil, R.H. Pletcher, Computational fluid mechanics and heat transfer, Hemisphere publishing corporation, New York (USA), 1984

[18] J.P. Nougier, Méthodes de calcul numérique, Masson, $3^{\mathrm{e}}$ édition, Paris, 1987

Retrouvez nos articles sur le site : www.edpsciences.org/meca 